
allemande

49-1 | 2017

Berlin 1957-1994

\title{
«Kreuzberg 36 » se révolte : occupations d'immeubles et luttes urbaines à Berlin (années 1970 et 1980)
}

\section{Patrick Farges}

\section{(2) OpenEdition}

\section{Journals}

Édition électronique

URL : https://journals.openedition.org/allemagne/527

DOI : 10.4000/allemagne.527

ISSN : 2605-7913

Éditeur

Société d'études allemandes

\section{Édition imprimée}

Date de publication : 16 juin 2017

Pagination : $99-116$

ISSN : 0035-0974

\section{Référence électronique}

Patrick Farges, « « Kreuzberg 36 » se révolte : occupations d'immeubles et luttes urbaines à Berlin (années 1970 et 1980) », Revue d'Allemagne et des pays de lanque allemande [En ligne], 49-1 | 2017, mis en ligne le 16 juin 2018, consulté le 02 juin 2022. URL : http://journals.openedition.org/allemagne/527 ; DOI : https://doi.org/10.4000/allemagne.527 


\section{«Kreuzberg 36 » se révolte: occupations d'immeubles et luttes urbaines à Berlin (années 1970 et 1980)*}

\section{- Patrick Farges**}

À la fin des années 1960, en réaction à l'urbanisme fonctionnaliste, naît une pensée critique qui souligne que l'organisation spatiale est un élément déterminant de la vie sociale. La mise en place d'une planification urbaine dans l'après-guerre en Europe de l'Ouest avait en effet consacré un pouvoir expert et technocratique, contre lequel il s'agissait de réhabiliter les pulsations urbaines ${ }^{(1)}$. L'urbain est repensé comme un ensemble d'espaces multiples, produits par des pratiques et usages sociaux soumis à différents rapports de pouvoir ${ }^{(2)}$ : dès lors, il devient possible de penser la «spatialisation» (Verräumlichung) des rapports sociaux ${ }^{(3)}$. Nés dans le sillage des mouvements étudiants et de la «nouvelle gauche», les «nouveaux mouvements sociaux» en RFA se sont saisis de ces réflexions. Ils partageaient, outre une sociologie et des modes de vie communs, un répertoire politique, des formes d'action en rupture avec la mobilisation ouvrière, un langage radical, doublé d'une attention portée à l'efficacité rhétorique.

Parmi ces groupes - groupes d'entraide, centres de soins alternatifs, mouvements des minorités sexuelles, mouvements «autonomes »-il y a le mouvement d'occupation d'immeubles (Hausbesetzerbewegung) ${ }^{(4)}$. La question de l'habiter y croise les nouvelles mobilisations politiques et sociales, à l'occasion des luttes relatives à la préservation de l’habitat en centre-ville. Pour les personnes mobilisées, il s'agissait de créer des «Frei-

* Je remercie les documentalistes de la Zentral- und Landesbibliothek Berlin (site de Breite Straße) pour leur aide précieuse ainsi qu'Antoine Fleury et Valérie Carré pour leurs relectures attentives.

** Maître de conférences HDR, Département d'études germaniques, Université Sorbonne Nouvelle Paris 3.

1 Pour la France, ce « tournant» est incarné par Henri Lefebvre (Le Droit à la ville [1968], suivi d'Espace et politique, Paris, Anthropos, 1972).

2 Henri Lefebvre, «La production de l'espace», L'Homme et la société, 31/1 (1974), p. 15-32.

3 Martina Löw, Raumsoziologie, Francfort-sur-le-Main, Suhrkamp, 2001.

4 Roland Rотн, Dieter Rucht (éd.), Die sozialen Bewegungen in Deutschland seit 1945. Ein Handbuch, Francfort-sur-le-Main, Campus, 2008. 
räume», c'est-à-dire des espaces d'intérêt collectif et d'usage public, libres mais aussi libérés de certains rapports de domination: cafés alternatifs (Szenekneipen), centres d'information politisés (Infoläden), etc. Le cas de Berlin-Ouest est particulièrement parlant, notamment parce qu'on y trouve une "Szene» alternative de gauche, où se construisent des logiques subculturelles collectives, qui contribuent à «faire» la ville. En retour, des identités collectives urbaines se sont créées par des mobilisations au quotidien, rattachées à des lieux précis ${ }^{(5)}$. C'est ce qui nous intéresse ici.

Une première manière d'aborder le phénomène est de s'intéresser aux slogans brandis lors de manifestations ou sur les façades. Ils offrent un mélange de radicalité politique assumée, d'utopie urbaine à construire, de références historiques et culturelles que l'on trouve dans la "génération 68 », et d'inventivité linguistique propre aux nouveaux mouvements sociaux ${ }^{(6)}$ :

«Friede den Besetzern, Kampf den Besitzern!» ${ }^{(7)}$

«Lieber instandbesetzen als kaputtbesitzen!

Kaputte Stadt retten.

Legal - illegal - scheißegal.

Des Spekulanten Brot ist Wohnungsnot ${ }^{(8)}$.

«Der Kiez gehört denen, die hier leben! [...] K36 lebt solange wir uns wehren » ${ }^{(9)}$.

«Wir haben nichts zu verlieren als unsere Angst.

Wir brauchen Räume, die ein herrschaftsfreies Leben zumindest vorstellbar machen ${ }^{(10)}$.

«Über die Provokation der Absa(h)nierungspolitik rein in die aufgelassenen Häuser!

Geschichte? Planung? - Scheiß drauf, wir sind jetzt drin! " ${ }^{(1)}$

«Kein Abriß unter dieser Nummer» ${ }^{(12)}$.

Les slogans produits en l'espace de quelques années dans les collectifs de Kreuzberg frappent par leur force rhétorique. Ils mettent en avant les «braconnages» façonnant l'espace public urbain à l'époque ${ }^{(13)}$ et permettent un survol sémiotique de cette subculture militante. Les nouveaux mouvements sociaux, tels qu'ils se sont exprimés à Berlin-Kreuzberg à travers les occupations et la création d'espaces alternatifs, allient

5 Cf. Tatiana Golova, Räume kollektiver Identität. Raumproduktion in der "linken Szene" in Berlin, Bielefeld, transcript, 2011.

6 On retrouve cette même inventivité au sein du mouvement de mobilisation anti-nucléaire ou du mouvement féministe.

7 Cf. Arlette Moser, Wolfgang Albrecht, Peter Otт, Instandbesetzungen in Kreuzberg: Friede den Besetzern, Kampf den Besitzern!, Berlin, Sozialistische Einheitspartei Westberlins KV Kreuzberg, 1981, s. p.

8 Slogans extraits de Lothar Schмid, Häuserkampf im Berlin der 1980er, Berlin, Berlin Story Verlag, 2013.

9 A.G. Grauwacke, Autonomie in Bewegung. Aus den ersten 23 Jahren, Berlin/Hambourg, Assoziation A, 2008, p. 79.

10 Stefan Aust, Sabine Rosenbladt (éd.), Hausbesetzer. Wofür sie kämpfen, wie sie leben und wie sie leben wollen, Hambourg, Hoffmann und Campe, 1981, p. 7 sq.

11 Boris Penth, Günter Franzen, Last Exit - Punk: Leben im toten Herz der Städte, Reinbek, Rowohlt Taschenbuch, 1982, p. 147.

12 Cf. Bernd Laurisch, Kein Abriß unter dieser Nummer: 2 Jahre Instandbesetzung in der Cuvrystraße in Berlin-Kreuzberg, Gießen, Anabas, 1981.

13 Sur le «braconnage», cf. Michel de Certeau, L’Invention du quotidien, vol. 1 : Arts de faire, éd. L. Giard, Paris, Gallimard, 1990. 
d'une part des revendications issues de la démocratie radicale et de la participation citoyenne directe et, d'autre part, une volonté de transformer le quotidien par des modes de vie anticonformistes. Il s'agit là de politiques «de la première personne» ${ }^{(14)}$, où l'échelle micro joue un rôle primordial. La question des modalités de l'action militante y est déterminante, en particulier celle de l'utilisation ou non de la violence.

Dans le même temps, le dynamisme, la jeunesse, l'inventivité voire l'aspect insurrectionnel de ce milieu alternatif remettaient largement en question, dans le sillage des revendications intellectuelles de l'après-1968, l'ordre socio-économique ainsi que le consensus politique hérités. Comme l'exprime un témoin de l'époque:

«Hausbesetzungen verunsichern und provozieren das politische Establishment und die mehr oder minder konservative Mehrheit älterer Westdeutscher. Kein Zweifel: Hausbesetzungen sind keineswegs isolierte Aktionen wohnungssuchender Vagabunden, sondern konkrete Demonstrationsversuche und Kristallisationspunkte einer handlungsorientierten Alternativbewegung, die nicht in papiernen Programmen und Postulaten stecken bleiben will» ${ }^{(15)}$.

Nous proposons ici de rappeler l'histoire d'un mouvement à l'échelle d'un quartier sur quelques années. Ce sera aussi l'occasion d'aborder à une échelle locale et décentrée l'histoire des politiques urbaines. Dans un premier temps, nous rappellerons certains aspects de la politique de rénovation urbaine avant le milieu des années 1970. Ensuite, nous présenterons l'identité du quartier qui nous intéresse ici: Kreuzberg SO 36. Enfin, nous ferons la chronique (entre 1979 et 1982) de la mobilisation des «occupa-rénovateurs» (Instandbesetzer) et de leur ancrage local.

\section{Habitat et politiques de rénovation urbaine}

L'histoire des mobilisations urbaines à l'échelle d'un quartier s'articule avec un contexte plus large et multi-scalaire, qui caractérise largement l'histoire de Berlin dans l'après-guerre: Berlin est observée à l'échelle internationale (et interallemande) dans le cadre d'une concurrence des systèmes qui influe sur la politique urbaine; à l'échelle fédérale, Berlin-Ouest constitue un foyer de contestation depuis l'après-1968; à l'échelle locale enfin, plusieurs plans d'aménagement se sont succédé, produisant à chaque fois des effets sociaux ${ }^{(16)}$. Le contexte qui nous intéresse ici est celui de la décennie qui a suivi l'accord quadripartite sur le statut de Berlin (1971), qui a eu pour effet collatéral de détourner l'attention internationale ${ }^{(17)}$, et de relâcher quelque peu les injonctions contradictoires pesant sur la politique urbaine. Après 1971, Berlin a moins eu à jouer le rôle de «ville-front» $(\text { Frontstadt })^{(18)}$. Car il est vrai que dans les premières

14 Roland Roth, Dieter Rucht, "Autonomie und die Politik der ersten Person ", in: Roth/Rucht (éd.), Die sozialen Bewegungen (note 4), p. 459 sq.

15 Karl-Michael Kuntz, «Spontis, Schlaffis und Chaoten. Psychologische und politische Perspektiven der neuen Jugendbewegung», in: Aust/Rosenbladt (éd.), Hausbesetzer (note 10), p. 193.

16 Sur l'imbrication des différentes échelles, cf. Dirk Rotenberg, Berliner Demokratie zwischen Existenzsicherung und Machtwechsel 1971-1981, Berlin, Haude \& Spener, 1995, p. 1 sq.

17 Arnulf Baring évoquait une «Außenfixierung». Arnulf BARInG, «Die Rolle Berlins nach dem Zweiten Weltkrieg», Jahrbuch für die Geschichte Mittel- und Ostdeutschlands, vol. 34 (1985), p. 28.

18 Cf. Thomas Flemming, Berlin im Kalten Krieg. Der Kampf um die geteilte Stadt, Berlin, be.bra Verlag, 2008. 
années de l'après-guerre, tant Berlin-Ouest que Berlin-Est avaient fait l'objet de projets urbanistiques spectaculaires de reconstruction ${ }^{(19)}$. Ces expérimentations ont conduit à d'importantes destructions de la substance héritée de la Gründerzeit ${ }^{(20)}$. À l'Ouest, cela s'est fait sous l'égide d'un Sénat où les sociaux-démocrates ont la majorité absolue entre 1958 et 1975, puis une majorité relative, en coalition avec les libéraux, dans la seconde moitié des années 1970. La politique en matière de logement, de transports, d'éducation et en matière sociale s'est largement confondue avec les options prises localement par le SPD, en accord avec les sociétés en régie directe (Berliner Eigenbetriebe).

Le premier grand plan de rénovation urbaine à Berlin-Ouest, après la phase de reconstruction, a débuté dès les années 1960, avec une volonté de créer du logement social. Il s'agissait de répondre à l'une des traces de la guerre: la problématique de l'insalubrité de certains îlots. Des zones (Sanierungsgebiete) sont alors définies. C'est à Wedding que sont expérimentés des procédés et techniques contestés par la suite, en raison de leur radicalité et de l'absence de concertation: la rénovation intégrale de grands périmètres urbains (Flächensanierung) par destruction totale (Totalabriss) ou partielle, notamment par le biais de l'évidement d'îlots (Blockentkernung). Ces procédés ont été perçus comme menant à des "coupes claires» dans le tissu urbain (Kahlschlagsanierung) ${ }^{(21)}$. Par ce biais, la priorité politique du Sénat, qui évoque même en 1964 le danger d'avoir des «slums» au cœur de la ville, est d'empêcher la constitution de quartiers en crise et d'îlots dégradés concentrant les problèmes. L'urbanisme d'alors est celui du «miracle économique» et il dévalorise le bâti ancien de centre-ville.

Parallèlement, ce qui correspond d'ailleurs à une évolution générale des métropoles européennes jusque dans les années 1980, la politique des transports est largement orientée vers une «ville adaptée à l'automobile» (autogerechte Stadt). Il est ainsi prévu de percer de grands axes autoroutiers (Stadtautobahnen). Or Kreuzberg est situé sur l'axe projeté de la "tangente sud»(Südtangente). De ce fait, une partie du quartier doit être transformée en échangeur ${ }^{(22)}$. Ces projets, en gestation depuis le milieu des années 1960, ont pris du retard, notamment parce que l'accord quadripartite de 1971, qui scelle la division de Berlin, a nécessité qu'ils soient adaptés, sans toutefois être abandonnés. Le résultat en est que dans les quartiers concernés, l'investissement dans la rénovation du parc immobilier recule, les propriétaires spéculant sur les grands travaux à venir. Cela produit des effets cumulatifs et des évictions d'habitants vers la périphérie, où des grands ensembles ont vu le jour depuis les années 1960, par exemple Märkisches Viertel ou Gropiusstadt. C'est cette «question sociale» qui mettra le feu aux poudres.

19 Cf. Theo Winters, Niklas Fluß, «Überblick über die Stadterneuerung im geteilten Berlin », in : Günter Schlusche, Verena Pfeiffer-Kloss, Gabi Dolff-Bonekämper, Axel Klausmeier (éd.), Stadtentwicklung im doppelten Berlin. Zeitgenossenschaften und Erinnerungsorte, Berlin, Christoph Links Verlag, 2014, p. 176-189.

20 Cf. Harald Bodenschatz, Cordelia Polinna, «Ein halbes Jahrhundert Stadterneuerung in Berlin», in: Schlusche et al. (éd.), ibid., p. 206-213.

21 Cf. Heinrich Suhr, «Stadterneuerung in West-Berlin am Beispiel Ackerstrasse Nord», in: SCHLUSCHE et al. (éd.), ibid., p. 98-109.

22 Cf. Udo Dittfurth, "Verkehrsplanung in West-Berlin. Ein Bericht aus dem ideologischen Sektor", in: Schlusche et al. (éd.), ibid., p. 226-241. 
Dès la fin des années 1960 et le début des années 1970, des voix se font entendre, qui dénoncent la brutalité de la rénovation urbaine et réclament des formes plus douces (behutsam) respectant les cadres de vie locaux. En 1975, dans le cadre de l'année européenne du patrimoine architectural, qui débouche sur la signature d'une Charte, de gros efforts sont déployés dans l'ensemble des pays européens pour sensibiliser aux valeurs culturelles, sociales et économiques des monuments et des sites hérités du passé. Cela conduit pour Berlin à une lente remise en question de la rénovation, initiée à la fin du mois d'avril 1976 par la tenue, dans le cadre du Conseil de l'Europe, d'une Conférence sur la conservation du patrimoine architectural. Émerge alors l'idée de «réparation urbaine» (Reparatur) voire de «reconstruction» (Rekonstruktion) ${ }^{(23)}$.

En 1974, le portage des projets de rénovation urbaine est modifié: le Sénat décide de faire entrer des sociétés privées dans les projets, justifiant sa décision par la taille des zones à rénover ${ }^{(24)}$. Cette décision est vivement critiquée par les habitants. Par ailleurs, le système des grandes sociétés de logement (Wohnungsbaugesellschaften) était taillé pour des rénovations intégrales de grande ampleur et non pour la concertation à petite échelle. Dans ce contexte, personne - ni les sociétés de logement, ni les nouveaux acteurs privés, ni le Sénat - ne voit l'intérêt d'expérimenter de nouveaux modèles participatifs et les habitants perturbent plutôt les rouages d'une politique bien huilée. Harry Ristock, sénateur au logement, a beau déclarer en 1978 que la rénovation urbaine garantira dorénavant continuité historique et préservation des structures ${ }^{(25)}$, le système politique en place est difficile à changer. Hartmut Häußermann et Andreas Kapphan font ainsi le constat amer que c'est bien la politique en matière d'urbanisme qui a produit des zones urbaines sensibles, en négligeant le tissu social de certains quartiers, au premier rang desquels Kreuzberg SO $36^{(26)}$.

\section{Kreuzberg: la poudrière de Berlin?}

L'arrondissement de Kreuzberg est l'héritier du faubourg de la Luisenstadt, qui a radicalement changé de visage durant la révolution industrielle. Le quartier est constitué à la fois d'immeubles d'habitation, d'ateliers d'artisans et de commerces, créant ce «mélange» (Kreuzberger Mischung) typique des faubourgs industriels ${ }^{(27)}$, marqué en particulier par l'habitat socialement et fonctionnellement dense des «casernes

23 Cf. IBA (éd.), Idee Prozeß Ergebnis - Die Reparatur und Rekonstruktion der Stadt, Berlin, Fröhlich \& Kaufmann, 1984. Voir aussi Harald Bodenschatz, Volker Heise, Jochen Korfmacher (éd.), Schlu $\beta$ mit Zerstörung? Stadterneuerung und städtische Opposition in West-Berlin, Amsterdam und London, Gießen, Anabas, 1983.

24 Andreas Suttner, 'Beton brennt': Hausbesetzer und Selbstverwaltung in Berlin, Wien und Zürich der 80er Jahre, Münster, Lit-Verlag, 2011, p. 112.

25 «Die Sanierung kann heute nicht mehr als Flächensanierung, sondern muß als eine unsere gewachsenen Strukturen erhaltende Stadterneuerung verstanden werden. » Cité dans Harald BodensCHATZ, Platz frei für das neue Berlin! Geschichte der Stadterneuerung seit 1871, Berlin, Transit, 1987, p. 190.

26 Hartmut HäUßermann, Andreas Kapphan, Berlin. Von einer geteilten zur gespaltenen Stadt? Sozialräumlicher Wandel seit 1990, Opladen, Leske + Budrich, 2000, p. 80-81.

27 Cf. Karl-Heinz Fiebig, Dieter Hoffmann-Axthelm, Eberhard Knödler-Bunte (éd.), Kreuzberger Mischung. Die innerstädtische Verflechtung von Architektur, Kultur und Gewerbe, Berlin, Verlag Ästhetik und Kommunikation, 1984; Erika Hausmann, Clarissa Soltendiek, Von der Wiese zum Baublock: Studie zur Entwicklungsgeschichte der Kreuzberger Mischung, Berlin, Publica, 1986. 
locatives» (Mietskasernen). C'est un quartier à l'identité ouvrière, en particulier dans sa partie nord-est. Cette dernière, qui appartenait à la zone postale du «Sud-Est 36 » du Grand-Berlin (Südost 36 ou SO 36) a gardé le nom de «Kreuzberg SO 36 » ou « Kreuzberg 36 ", même après que l'indicatif 36 a définitivement disparu des codes postaux en 1993. Durant les bombardements alliés de la Seconde Guerre mondiale, la zone est durement touchée, et les «trous urbains» (Baulücken), tout comme les sites industriels à l'abandon sont une réalité visible. De plus, Kreuzberg 36 est enclavé: au nord et à l'est, il y a à partir de 1961 le Mur; à l'est et au sud, la limite est marquée par la Spree et le canal. Dans les années 1960, la zone de SO 36 autour de Kottbusser Tor, délimitée à l'est par la Manteuffelstraße, à l'ouest par l'Oranienplatz, au nord par la Naunynstraße et au sud par la Skalitzer Straße, est classée en "zone d'aménagement» (Sanierungsgebiet) et des "coupes claires» y sont prévues. Cette zone se trouve dans l'immédiat voisinage d'un autre micro-quartier particulièrement enclavé, autour de la Wrangelstraße ${ }^{(28)}$. C'est toute cette zone qui devient dans les années 1970 et 1980 un «point chaud».

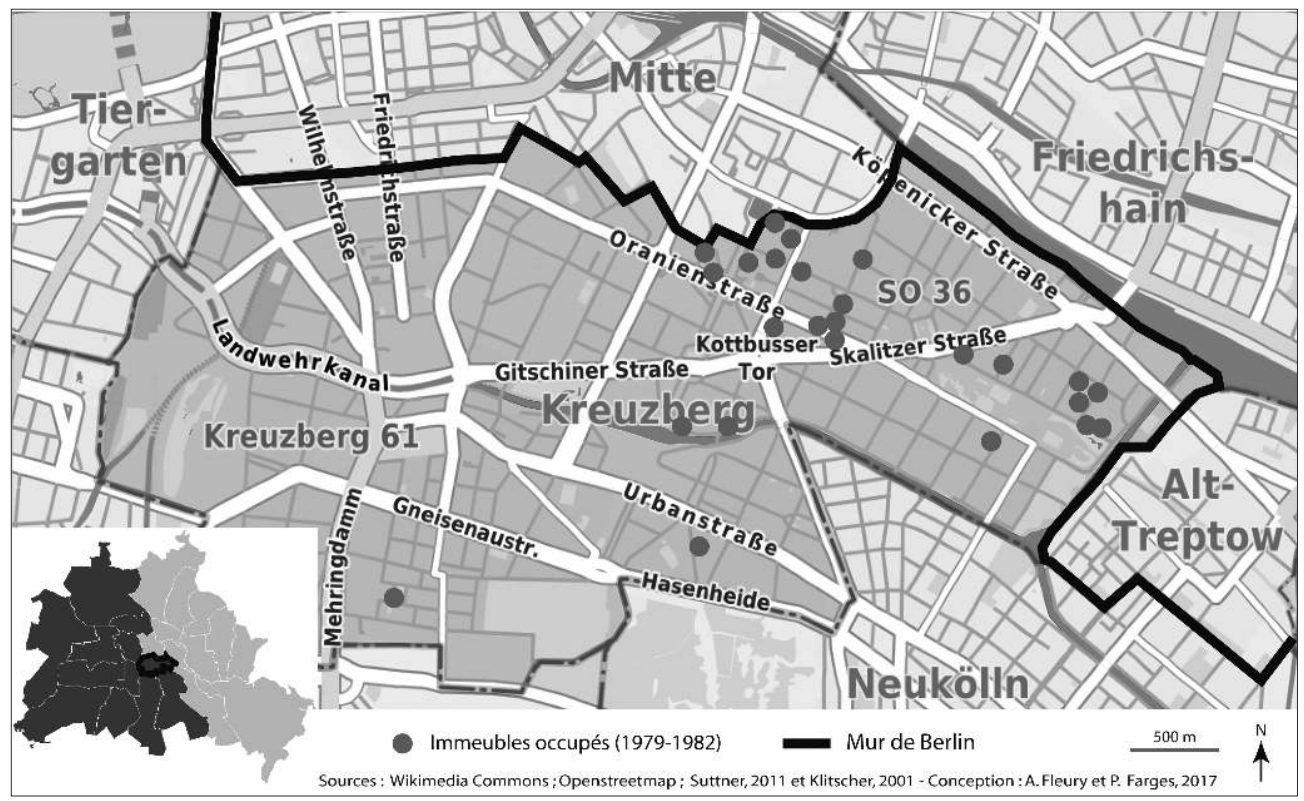

Alors qu'à Berlin-Ouest en général, la proportion de logements anciens et neufs est à peu près équilibrée à la fin des années 1970, le déséquilibre en faveur de l'ancien reste très fort à Kreuzberg. En raison de la faiblesse des loyers, liée à la décrépitude des bâtiments et au contrôle des loyers, SO 36 est devenu en quelques années un lieu de vie pour différentes populations au revenu limité: personnes âgées, étudiants, «alternatifs», populations d'origine immigrée, en particulier d'origine turque. On assiste donc à une nouvelle forme de «mélange kreuzbergeois», caractérisée par le

28 Cf. Hartmut HäUßermann, «Wohnen in Berlin. Die Entwicklung sozialräumlicher Strukturen », in: Werner Süss, Ralf Rytlewski (éd.), Berlin. Die Hauptstadt. Vergangenheit und Zukunft einer europäischen Metropole, Bonn, Bundeszentrale für politische Bildung, 1990, p. 468-501. 
multiculturalisme et une forte imprégnation par les cultures de jeunesse ${ }^{(29)}$. Si d'autres arrondissements de Berlin-Ouest sont également marqués par les (sub)cultures alternatives de l'après-1968 ${ }^{(30)}$, Kreuzberg l'est davantage: c'est aussi ce qui en fait l'attrait pour qui cherche à vivre différemment. Le sénateur berlinois en charge de la recherche à partir de 1977, Peter Glotz, en prend acte lorsqu'il évoque publiquement la coexistence de «deux cultures» parmi les jeunes: l'une «établie», l'autre «alternative» ${ }^{(31)}$. Kreuzberg 36 a ainsi développé une forte identité locale et constitue un «Kiez» berlinois, c'est-à-dire un quartier à l'identité marquée par des relations sociales de proximité et une dynamique culturelle propre ${ }^{(32)}$. Dans le contexte d'une décennie marquée par les nouveaux mouvements sociaux, SO 36 développe une économie alternative, des groupes d'entraide, des «ateliers d'histoire citoyenne»(Geschichtswerkstätten), et propose une alternative politique, puisque c'est le terreau qui voit naître la «Liste alternative», précurseur des Verts. L'éventail de l'engagement est large; il inclut une réflexion concrète sur l'habiter et le vivre-ensemble, qui dépasse les mobilisations «à cause unique» (single-issue-movements $\left.{ }^{(33)}\right)$. Comme le rappelle un témoin de l'époque:

«Für mich war es entscheidend, auch in einer größeren Gruppe - es sind also 20 bzw. 25 Leute meistens in solchen Häusern - zusammenzuleben und [...] das Leben in der Gruppe halt zu organisieren [und] auch Versuche zu unternehmen, außerhalb der üblichen Arbeiten oder Schule oder Universität ein Selbst zu finden, Projekte oder irgendwelche Selbsthilfegruppen oder irgendwelche Angelegenheiten oder auch nur mal in der Arbeit mit den anderen Mietern hier, halt wirklich einen Versuch von unten, so eine Politik zu machen und die sich nicht immer von oben aufsetzen zu lassen ${ }^{(34)}$.

En 1979-80, la crise du marché du travail accentue la nécessité de ces économies alternatives reposant sur l'entraide, le troc et la solidarité. «Faire Kiez» est donc intimement lié aux caractéristiques sociales, aux formes du logement (locatif) et du bâti (immeubles avec arrière-cours). À partir des années 1970, la pression immobilière augmente fortement, aussi en raison de l'augmentation du nombre des ménages individuels, qui passe de 44 à $53 \%$ au cours de la décennie. Pourtant, dans le même temps, la démographie de Berlin-Ouest est globalement en recul. Environ $40 \%$ des ménages ont un revenu faible, $15 \%$ sont au seuil de pauvreté. $80 \%$ des ménages dépensent un quart ou plus de leur revenu pour le loyer (hors charges locatives). La perspective d'une destruction ou d'une rénovation de logements anciens pour faire du neuf est particulièrement anxiogène pour toute une partie de la population: si le loyer plancher

29 Cf. Barbara LAng, Mythos Kreuzberg: Ethnographie eines Stadtteils (1961-1995), Francfort-sur-leMain, Campus, 1996.

30 Cf. Olaf Leitener (éd.), West-Berlin! Westberlin! Berlin (West)! Die Kultur - die Szene - die Politik. Erinnerungen an eine Teilstadt der 70er und 80er Jahre, Berlin, Schwarzkopf \& Schwarzkopf, 2002.

31 Cité dans D. Rotenberg, Berliner Demokratie (note 16), p. 394.

32 Sur l'importance de l'échelle du «Kiez», voir Ralf Rytlewski, «Berliner Politik: Zwischen Kiez und Stadtstaat », in: Süss/Rytlewski (éd.), Berlin (note 28), p. 295-329.

33 Stephen P. Turner, Gerard Delanty, «Social, Political, and Cultural Theory since the Sixties», in: S. Turner, G. Delanty (éd.), Routledge International Handbook of Contemporary Social and Political Theory, New York, Routledge, 2011, p. 1-30.

34 Walter Hornstein, Unsere Jugend - Über Liebe, Arbeit und Politik, Weinheim/Bâle, Beltz, 1982, p. 229-230. 
dans le logement social neuf est d'environ $6 \mathrm{DM} / \mathrm{m}^{2}$, il se situe entre 1,80 et $4 \mathrm{DM} / \mathrm{m}^{2}$ dans l'ancien non rénové ${ }^{(35)}$.

La «tradition» des occupations d'immeubles à Kreuzberg débute au début des années 1970. En juillet 1971, des jeunes investissent deux étages d'une usine désaffectée, au 13 de la Mariannenstraße, pour y instaurer un foyer culturel; ils sont rapidement délogés par la police. Dès décembre, l’hôpital de Béthanie, fermé en 1970, est occupé par 300 étudiants venus de l'Université technique. Au bout de trois semaines de négociations, les occupants obtiennent un bail provisoire ${ }^{(36)}$. Des organes de concertation et de participation des habitants existent également dès 1974 à Kreuzberg, avec la mise en place d'un conseil de quartier à Kottbusser Tor, qui réclame d'être consulté à propos du grand ensemble "Neues Kreuzberger Zentrum», décidé en $1971^{(37)}$. La résistance des habitants conduit à un ralentissement des travaux. En 1977, un appel à projet, «Stratégies pour Kreuzberg», est lancé, qui conduit à la structuration de divers conseils de quartiers en une «initiative citoyenne», la "Bürgerinitiative SO 36 e.V.» (ou BI SO 36), qui démarche individuellement les habitants ${ }^{(38)}$. En 1978, poursuivant et élargissant les réflexions des «Stratégies pour Kreuzberg», le Sénat décide d'organiser l'Exposition internationale d'architecture (Internationale Bauausstellung, IBA), afin de répondre à la question de la «reconquête du centre-ville comme lieu d'habitation» (Wiedergewinnung der Innenstadt als Wohnort).

Il s'agit de proposer des projets de "rénovation urbaine douce» (behutsame Stadterneuerung) et BI SO 36 est reconnu comme interlocuteur légitime. En 1977, la coalition sociale-libérale a également proposé un nouveau "modèle de participation» (Modell der Betroffenenmitwirkung), où les habitants choisissent leurs représentants ${ }^{(39)}$. Mais la mise en place des structures de concertation patine et le nombre de mécontents grossit. Le processus lancé par l'IBA prendra environ dix ans avant de donner des résultats concrets. La mobilisation "par le bas» contribue de manière déterminante à l'émergence de nouveaux acteurs locaux et à une prise de conscience collective, qui finit par se retourner contre la politique de la ville. Un pamphlet de l'époque, intitulé «zones libres» (rechtsfreie Räume), rappelle les enjeux de la mobilisation:

«In diesem Stadtteil sammelt sich [die "Szene"], in diesem Kreuzberg, das vor 20 Jahren die erste Sanierungswelle über sich ergehen lassen mußte, deren betongewordenes Denkmal das Märkische Viertel und die Wunden im Stadtbild des alten Kreuzbergs sind - eine Sanierung, deren Sinn nicht nur in den hohen Profiten der Bauwirtschaft lag, sondern zugleich auch in der Zerschlagung eines alten Arbeiterviertels, eines "Kiezes", in dem es noch Reste von Zugehörigkeit gab» ${ }^{(40)}$.

Cf. Horst Riese, «Wohnen in Berlin», in: Ingrid Müller-MünCh, Wolfgang Prosinger, Sabine Rosenbladt, Linda Stibler et al., Besetzung - weil das Wünschen nicht geholfen hat. Köln, Freiburg, Gorleben, Zürich und Berlin, Reinbek, Rowohlt, 1981, p. 94-107.

36 A. SutTner, 'Beton brennt' (note 24), p. 114-115.

37 Sophie Perl, Erik Steffen (éd.), Abriss und Aufbruch am Kottbusser Tor 1945-2015, Berlin, FHXB Museum, 2015.

38 A. Suttner, 'Beton brennt' (note 24), p. 113.

39 H. Bodenschatz, Platz frei (note 25), p. 195.

40 «Rechtsfreie Räume», in: Aust/Rosenbladt, Hausbesetzer (note 10), p. 152. 
La deuxième moitié des années 1970 annonce la fin de la «vague rouge» (socialdémocrate) à Berlin-Ouest. Entre 1963 et 1975, le SPD, usé et déconsidéré, a perdu près de 20 \% de voix. En mai 1977, le maire Klaus Schütz démissionne en raison d'un scandale financier impliquant un membre du Sénat. Il est remplacé par Dietrich Stobbe, fils spirituel de Willy Brandt. Mais le SPD s'est coupé d'une partie de la population, jeune ou alternative, qui aspire, dans l'«après-1968», à des changements plus radicaux.

\section{Le mouvement social des «occupa-rénovateurs» (Instandbesetzer) : chronique d'une mobilisation (1979-1982)}

Le mouvement d'occupation qui débute en 1979-80 constitue une mobilisation protestataire spectaculaire contre la politique de rénovation urbaine, les failles du marché locatif, les "coupes claires» occasionnées dans le tissu urbain et la politique de dédensification des cœurs d'îlots. Le changement de paradigme sous l'égide du sénateur au Logement, Harry Ristock, qui prône une politique de «réparation urbaine» (Stadtreparatur) n'y change rien. Les premières «rénovations douces» ont en réalité produit des logements de standing, qui renforcent la pression locative à la hausse. Certains propriétaires n'hésitent pas à accélérer la dégradation du bâti ancien en cassant les fenêtres ou en abîmant les toitures ${ }^{(41)}$. On aboutit à un paradoxe: alors même qu'au tournant des années 1980, plusieurs dizaines de milliers d'unités de logements et des centaines d'immeubles à Berlin-Ouest sont vides, il y a pénurie de logements ${ }^{(42)}$. Le marché est segmenté entre l'ancien et le neuf, et la spéculation immobilière pérennise les logements vides. C'est de cette contradiction que naît le mouvement des occupants.

Le 3 février 1979, l'initiative citoyenne BI SO 36 investit illégalement deux appartements situés dans deux immeubles (l'un Görlitzer Straße, l'autre Lübbenerstraße) appartenant à la société de logements publique $\mathrm{BeWoGe} \mathrm{e}^{(43)}$. Cela marque le début d'une série d'occupations dans le quartier. Un mois plus tard, c'est l'étage d'un immeuble industriel, au 33 de la Waldemarstraße, qui est occupé par de jeunes chômeurs réclamant des lieux «de vie et de travail». En mai, la police empêche de justesse l'occupation de l'immeuble situé au numéro 8, Fraenkelufer. Début septembre 1979, l'immeuble situé au numéro 9, Leuschnerdamm est intégralement occupé. Dans la Cuvrystraße, aux numéros 20-23 et 25-27, des destructions sont prévues, afin de permettre la construction d'immeubles neufs. Le 26 novembre 1979, des membres de BI SO 36 occupent trois appartements au numéro 25. Mi-décembre, la société immobilière BeWoGe procède à une destruction partielle du numéro 27. C'est à cette occasion que le mouvement prend son nom: il s'appellera «mouvement d'occupa-rénovation » (Instandbesetzer-Bewegung), qui prône la rénovation douce et respectueuse du bâti (Instandsetzung) au moyen d'une occupation illégale (Besetzung). La logique première

41 Cf. Jacqueline Klein, Sabine Porn, «Instandbesetzen», in: Müller-MünCH et al., Besetzung (note 35), p. 124.

42 On estime qu'en 1978, plus de 27000 logements sont vides à Berlin-Ouest. Début 1981, il en reste encore plus de 10000 . De même, environ 850 immeubles sont entièrement délaissés. Voir A. SuTtNER, 'Beton brennt' (note 24), p. 121.

43 Sur la chronologie des événements à Kreuzberg 36, voir Jörg KLitscher, «Der Häuserkampf in Kreuzberg», Berlinische Monatsschrift, nº 6 (2001), p. 150-154. 
vise toutefois à obtenir des contrats de location en bonne et due forme. Lorsque les premières régularisations ont lieu - signe que la stratégie est payante -, les occupations s'accélèrent.

En janvier 1980, douze appartements sont occupés dans divers immeubles de la Mariannenstraße, afin d'empêcher une revalorisation. Au même moment, les occuparénovateurs investissent les adresses suivantes: Heinrichplatz 8, Naunynstraße 77-79, Leuschnerdamm 37-39. Suivent en mars 1980: Luckauer Straße 3 et Mariannenstraße 48. Le nombre d'unités occupées oblige le mouvement à se structurer. Le 28 mars 1980, des représentants de divers immeubles occupés dans le quartier SO 36 se réunissent dans la Cuvrystraße et créent le «Conseil des occupants K36» (Besetzerrat K36), organe de représentation. Dorénavant, les rencontres seront hebdomadaires, une stratégie d'action commune est mise en place ainsi qu'une politique de communication externe. Le 29 mai 1980, alors que le collectif occupe un immeuble voué à la destruction au numéro 56, Wrangelstraße, la police intervient. En représailles, des militants se rendent dans la partie plus cossue de Kreuzberg, "Kreuzberg 61 », et occupent un immeuble de la société berlinoise de logement GeWoBAG situé au numéro 3, Chamissoplatz, qui abritait déjà un "bureau d'aide aux locataires" (Mieterladen). Le 4 juin, la police intervient pour déloger les occupants ${ }^{(44)}$.

Dès lors se met en place un jeu du chat et de la souris avec la police, dans le contexte d'une méfiance des milieux alternatifs envers les forces de l'ordre, héritée de l'état d'urgence en 1968 et du climat de l'«automne allemand» de 1977. Dans le même temps, il y a un flou juridique concernant l'occupation d'immeubles. En l'absence d'une loi interdisant explicitement l'occupation, la police a recours au droit pénal et civil, par exemple aux articles 123 («violation de domicile»), 242 («vols et agressions»), 248 ("consommation illégale d'électricité»), 303 ("dommages à la propriété ») voire, dans le cas d'un accès entravé à l'immeuble, l'article 129a ("soupçon d'association criminelle ou terroriste») ! En juin 1980, de nouveaux immeubles sont occupés : le numéro 6, Adalbertstraße, et le numéro 29, Fichtestraße. Parmi les personnes qui investissent ce dernier bâtiment, il y a le jeune député berlinois Walter Momper, membre du SPD et futur maire-gouverneur (1989-1991), qui allait plus tard, en novembre 1990, ordonner l'expulsion des squatteurs de la Mainzer Straße à Friedrichshain.

Le 5 juillet 1980, les «Indiens dans la ville» (Stadtindianer), micro-mouvement de gauche radicale, occupent l'immeuble des numéros 44 et 45 , Oranienstraße, et fondent un lieu alternatif mythique: le «Geronimo-Haus». Cela amorce une radicalisation croissante au cours de l'année 1980, qui conduit à une isolation progressive des membres du BI SO 36, avec leur politique de régularisation. La stratégie de médiation voulue par le Sénat de Dietrich Stobbe (coalition sociale-libérale), qui propose légalisations et relocalisations, n'est pas suivie ${ }^{(45)}$. Les revendications des occupa-rénovateurs évoluent: il ne s'agit plus seulement de «rénovation douce» et d'aménagement; il s'agit de créer des «zones franches» ou «libres» (Freiräume) au cœur de la ville,

44 Cf. Alf Bremer, Kreuzberg Chamissoplatz: Geschichte eines Berliner Sanierungsgebietes, Berlin, ProPolis-Verlag, 2007.

45 Le Parti libéral déclare pourtant que l'occupation peut être comprise comme une mesure d'entraide, certes radicale mais légitime. Voir A. SutTner, 'Beton brennt' (note 24), p. 130. 
où la vie s'organiserait collectivement. Le mouvement d'occupa-rénovation attire une jeunesse radicale et punk qui théorise la vie anti-bourgeoise et anticapitaliste, comme en témoigne l'extrait suivant:

«Die Grenzen zwischen Privatsphäre und öffentlichem Raum verschwimmen. Es geht nicht darum, sich schmuck und kuschelig einzurichten, sich zu der Abgrenzung zu der Welt draußen einen gemütlichen Schonraum zu schaffen. Man trifft sich in den Räumen wie man sich am Kottbusser Tor in Berlin Kreuzberg treffen würde, Boden wie sonst auf dem Bürgersteig, trägt drinnen wie draußen die gleichen Kleider » ${ }^{(46)}$.

Si les articles de presse (tant locale que fédérale) avaient jusque-là été plutôt bienveillants à l'égard des occupants, le ton change au cours de l'année 1980. De plus en plus fréquemment, les titres évoquent la radicalité, la violence voire l'action «terroriste». En réaction, le mouvement organise début octobre une «semaine d'information" publique afin de lutter contre la «diffamation médiatique» organisée notamment par le groupe Springer ${ }^{(47)}$. La semaine d'information se clôt le 10 octobre par une manifestation réunissant 1500 personnes, marquée par des échauffourées avec la police, par l'occupation du numéro 198, Oranienstraße, puis, deux semaines plus tard, du numéro 20, Admiralstraße. Le 8 novembre 1980, l'immeuble du numéro 50, Fraenkelufer, est occupé.

La radicalisation croissante s'accompagne d'une logique de diffusion de proche en proche, qui produit une insularisation (Verinselung) du "Wrangelkiez» dans le secteur Cuvrystraße/Wrangelstraße. Début novembre 1980, deux immeubles industriels (aux numéros 20 et 23, Cuvrystraße) s'ajoutent aux cinq immeubles déjà occupés dans le secteur, pour former un noyau cohérent, appelé "Kerngehäuse » ou "Cuvryland». L'îlot est dirigé par une association, "Kerngehäuse e.V.», et comporte une crèche parentale, un atelier de mécanique, un centre d'entraide médicale, une société alternative de construction, un magasin coopératif, une éolienne, une école de langue, le théâtre Ratibor ${ }^{(48)}$ ou encore un orchestre (le "Cuvryland Trümmer-Orkäster») ${ }^{(49)}$. Le 21 novembre 1980, c'est l'avant et l'arrière du numéro 36, Görlitzerstraße, qui est occupé : cette adresse sera pour de longues années connue sous le nom de «Villa Kunterbunt» (multicolore) sur rue, et de «Villa Dröhn» (vacarme) et «Villa Chaotica» dans l'arrière-cour. C'est au cours de cette période, fin 1980, que des habitants d'origine turque commencent également à recourir aux occupations en investissant deux immeubles vides de la Forster Straße. Le 5 janvier 1981, une «maison de sorcières» (Hexenhaus) exclusivement féminine est créée au numéro 5, Liegnitzer Straße. Le 27 février 1981, une "maison des soins" (Heilehaus) ouvre ses portes, composée d'un centre médical alternatif et d’une épicerie coopérative au numéro 36, Waldemarstraße.

46 Cité dans Monika Reimitz, «Drinnen und draußen. Vom Wohnen der Punks», in: Marlene Bock, Monika Reimitz, Hors-Eberhard Richter, Wolfgang Thiel, Hans-Jürgen Wirth (éd.), Zwischen Resignation und Gewalt - Jugendprotest in den achtziger Jahren, Opladen, Leske + Budrich, 1989, p. 103-104. Sur le lieu mythique de la culture punk qu'est le Club SO 36, voir Sub Opus 36 e.V. (SO 36) (éd.), SO 36. 1978 bis heute, Mayence, Ventil Verlag, 2016.

47 Cf. l'article de Valérie Robert dans le présent numéro.

48 Cf. http://www.ratibortheater.de, rubrique «Über uns» (dernière consultation: 17/02/2017).

49 Cf. la brochure d'information éditée par l'«Initiative Kerngehäuse Cuvrystraße»: Kerngehäuse. Gewerbehof Cuvrystraße 20/23. Leben und Arbeiten in SO 36, Berlin, 1980. 
En décembre 1980 commence la phase la plus tendue du phénomène d'occupa-rénovation, qui se poursuit jusqu'à l'été 1981, soit en pleine campagne pour les élections anticipées à la Chambre des députés. La presse se déchaîne, évoquant chaque semaine les débordements et la situation quasi-insurrectionnelle ${ }^{(50)}$. Le 12 décembre 1980, dix personnes tentent d'occuper l'immeuble vide du numéro 48, Fraenkelufer. La police procède à des arrestations. S'ensuit une bataille rangée entre manifestants, venus en nombre, et forces de l'ordre, qui se poursuit onze heures durant jusque tard dans la nuit. Les manifestants sont peu à peu refoulés vers Kottbusser Tor et Oranienplatz. $\mathrm{Au}$ passage, des vitrines de banques et de commerces sont saccagées. La tentative de médiation par le collectif des locataires de la Dresdner Straße ( Mieterinitiative Dresdner Straße», dirigée par Werner Orlowsky) et la BI SO 36 échoue. 58 personnes sont arrêtées (40 hommes, 18 femmes); 53 ont moins de trente ans. Le 15 décembre, une grande manifestation de soutien rassemble 15000 personnes. À partir de là, les revendications des occupa-rénovateurs incluent la libération des personnes arrêtées, au cri de "eins-zwei-drei, lasst die Leute frei». Comme d'autres mouvements de l'époque, les occupa-rénovateurs se radicalisent voire se militarisent, jusqu'à créer un corps alternatif d'infirmiers-secouristes arborant une croix rouge, des vestes en cuir et des casques de moto ${ }^{(51)}$. Ces modes d'action conduisent à un clivage irréconciliable entre, d'un côté, celles et ceux qui, organisés au sein d'associations, veulent «négocier» (Verhandler) et, de l'autre, celles et ceux qui ne le veulent pas (Nicht-Verhandler), qui peuvent en partie être rattachés à l'archipel complexe des mouvements radicaux «autonomes " ${ }^{(52)}$. Selon des témoins de l'époque, la ligne de fracture sépare les «idéalistes légalos» et les «fauteurs de trouble» $\left(\right.$ Krawallmacher ${ }^{(53)}$. Les «non-négociateurs autonomes» s'organisent en un «forum plénier» et préparent le «Congrès TUWAT» («Fais quelque chose» en berlinois) qui réunit à Berlin-Ouest 50000 personnes venues de toute l'Europe occidentale au mois d'août $1981^{(54)}$. L'un des autonomes témoigne:

«Bei uns Nichtverhandlern gibt es eine große Gruppe, die einfach in den Häusern bleiben will, solange es geht. Sie haben kein Interesse an Verhandlern, Instandbesetzern, Paten oder ähnlichem ${ }^{(55)}$.

Cette fracture recouvre des aspects idéologiques et politiques, mais également des caractéristiques sociologiques et générationnelles. Parmi les «négociateurs», il y a les membres des milieux alternatifs, les membres d'associations de quartier et des

50 Ce dont témoignent les volumineuses archives de presse à la Zentral- und Landesbibliothek Berlin (B-139 \& B-140, Presseschnitte, Hausbesetzungen).

51 Cf. «Im Fadenkreuz. Drei Mitglieder der Sanitäter-Gruppe über sich selbst », in: Aust/RosenBLADT, Hausbesetzer (note 10), p. 170-192.

52 Cf. la documentation réunie dans l'ouvrage anonyme: A. G. Grauwacke, Autonomie in Bewegung (note 9). Voir aussi Thomas Schultze, Almut Gross, Die Autonomen. Ursprünge, Entwicklung und Profil der autonomen Bewegung, Hambourg, Konkret Literatur Verlag, 1997; Jan SCHWARZMEIER, Die Autonomen zwischen Subkultur und sozialer Bewegung, Libri books on demand, 2001.

53 Michael Wieczorek, «Bullenparanoia und das Gefühl vom Paradies», in: Aust/Rosenbladt, Hausbesetzer (note 10), p. 106. Voir aussi Sabine RosenbladT, «Die 'Legalos' von Kreuzberg», in: ibid., p. 28-51.

54 En janvier 1978, le Congrès alternatif TUNIX («Ne fais rien») avait réuni 15000 personnes.

55 A. G. Grauwacke, Autonomie in Bewegung (note 9), p. 63. 
habitants de longue date, qui perçoivent les radicaux comme des personnes venues spécifiquement dans le quartier "pour en découdre». Rainer Milzkott, un «négociateur», décrit début 1981 le mouvement ainsi:

«Die Instandbesetzer können nicht als politische Gruppe definiert werden. Sie bezeichnen sich als "Stadtindianer", "Spontis", "Anarchisten" [...], "Aussteiger", "Freie Bürger", "Freaks" usw.»"(56).

La tension est renforcée par les aléas politiques que connaît Berlin-Ouest en 1981, l'«année des trois maires». Le 15 janvier, Dietrich Stobbe est contraint à la démission, en raison de l'affaire Garski, du nom de l'architecte dont la faillite coûte 100 millions de marks au contribuable. Hans-Jochen Vogel, figure d'envergure fédérale au SPD et ancien maire de Munich, devient maire durant cent jours, jusqu'aux élections anticipées. D’emblée, il cherche le dialogue et plaide pour qu'on distingue deux catégories d'occupa-rénovateurs: les personnes impliquées dans les problèmes sociaux et urbains, et les casseurs. La simple "violation de domicile» (Hausfriedensbruch) sans dégradation ni détérioration est tolérée. En guise de mea culpa politique, il met en place fin janvier 1981 une "Commission d'enquête sur les manquements en matière de rénovation et de modernisation urbaine et sur le retour à la paix sociale» et prend des mesures immédiates, chiffrées à 20 millions de marks, afin de financer des projets alternatifs. La responsabilité du Sénat concernant le problème des logements vacants et la destruction du tissu social est donc officiellement reconnue ${ }^{(57)}$. Le maire-gouverneur formule aussi en mars 1981 la politique de la «ligne berlinoise de la raison» (Berliner Linie der Vernunft), entérinée par son successeur: toute nouvelle occupation se soldera par une évacuation sous 24 heures; en contrepartie, les immeubles et appartements déjà occupés ne sont évacués que si le propriétaire porte plainte et s'engage à une rénovation rapide.

En réalité, cette «ligne» renforce les tensions. Une victoire de la CDU se dessine en effet, qui annonce une période plus intransigeante envers les occupants ${ }^{(58)}$. À l'hiver et au printemps, de nouveaux logements sont occupés presque quotidiennement, tant à Kreuzberg - où le mouvement proclame même une «République libre» - qu'ailleurs, par exemple autour de Winterfeldtplatz (Schöneberg) ou autour de Klausener Platz (Charlottenburg) ${ }^{(59)}$. En janvier 1981, 25 nouveaux immeubles sont occupés, soit autant en un mois que depuis le début du mouvement. Début février 1981, 10 nouveaux immeubles sont occupés. À la fin du mois de février, le mouvement revendique 100 immeubles occupés dans tout Berlin-Ouest. En mars 1981, 123 immeubles et deux églises sont occupés. Le mouvement s'accélère: au cours de la mandature de HansJochen Vogel, 171 nouvelles occupations ont lieu; 26 immeubles sont évacués par les occupants et 17 immeubles par la police. Les interventions policières se font de plus en

56 Cité dans A. Suttner, 'Beton brennt' (note 24), p. 138.

57 Cf. Heidede Becker, Jochen Schulz zur Wiesch (éd.), Sanierungsfolgen. Eine Wirkungsanalyse von Sanierungsmaßnahmen in Berlin, Stuttgart, Deutscher Gemeindeverlag, 1982.

58 Bernd Sonnewald, Jürgen Raabe-Zimmermann, Die Berliner Linie und die Hausbesetzer-Szene, Berlin, Berlin-Verlag Spitz, 1983.

59 Pour l'évolution des occupations, voir les cartes interactives sur le site: http://berlin-besetzt.de (dernière consultation: 15.02 .2017$)$. 
plus spectaculaires, comme lorsque, le 7 avril 1981, les 132 membres du «Conseil des occupants » de l'immeuble du numéro 3, Luckauer Straße, sont arrêtés, dont le propre fils du préfet de police Klaus Hübner. L’escalade paraît inévitable et les échauffourées se multiplient. À la mi-mai 1981, 169 immeubles sont occupés dans Berlin-Ouest, dont 80 à Kreuzberg uniquement. À l'occasion de la campagne électorale, des pancartes s'affichent sur les immeubles occupés: "Statt Wohnungspolitik plant die CDU den Bürgerkrieg!»; «Gegen Wohnungsnot, Abriß, Beton und Spekulation!» La "guerre des affiches» (Lappenkrieg) recommencera d'ailleurs de plus belle lors de la visite officielle du président américain, Ronald Reagan, en juin 1982: les slogans s'afficheront sur les façades jusqu'à ce que la police les fasse retirer.

La victoire de la CDU aux élections du 10 mai 1981 constitue une rupture politique importante. Le SPD essuie une défaite cinglante qui met fin à 35 années de pouvoir. Les élections marquent l'entrée à la Chambre des députés de la «Liste alternative», qui a obtenu 7,2 \% des suffrages et 12 députés. Cette liste, qui se définit comme porte-parole des occupa-rénovateurs (réclamant par exemple la relaxe immédiate des manifestants arrêtés) et comme son moyen d'accéder aux institutions ${ }^{(60)}$, a réussi à fédérer différentes énergies en fournissant au fil des mois un appui logistique au mouvement et en cultivant une identité anti-establishment. C'est d'ailleurs au titre de la "Liste alternative» que Werner Orlowsky (sans étiquette politique), représentant des habitants de Dresdner Straße, est nommé conseiller au Logement de Kreuzberg. Les craintes au sujet de la politique chrétienne-démocrate sont nombreuses: en mars, le congrès fédéral du parti, réuni à Mannheim, a voté la dérégulation du marché des logements sociaux. Pourtant, la politique envers les occupa-rénovateurs menée par le Sénat minoritaire (CDU), avec à sa tête Richard von Weizsäcker, est plutôt marquée par une certaine continuité. Dès février 1982, Weizsäcker avait d'ailleurs déclaré en interview ne pas vouloir «évacuer pour évacuer » ${ }^{(61)}$. Le nouveau sénateur au Logement, Ulrich Rastemborsky, annonce qu'il souhaite continuer le dialogue avec les occupa-rénovateurs. D’autres décisions vont dans le même sens, par exemple le fait que l'IBA (Internationale Bauausstellung) obtienne le statut d'aménageur à titre fiduciaire. Un an plus tard, en 1982, l'IBA devient organe de coordination auprès du sénateur au Logement afin de mettre en ouvre la politique de «rénovation douce» (behutsame Stadterneuerung), de concertation et de participation des habitants (Betroffenenbeteiligung).

Pour l'heure toutefois, le pouvoir décisionnel reste encore concentré dans les mains des grandes sociétés immobilières en régie directe, qui n'ont toujours pas intérêt à organiser la concertation. La question des occupations restera un problème aigu durant toute la mandature Weizsäcker. Le nouveau sénateur à l'Intérieur, Heinrich Lummer, qui signe les ordres d'expulsion, concentre la haine des occupa-rénovateurs. En réaction aux évacuations, les églises protestantes de Kreuzberg vont même jusqu’à se mobiliser pour parrainer des immeubles occupés ${ }^{(62)}$. Dès juin 1981, les affrontements

60 Selon la théorie politique de la complémentarité entre jambe d'appui (Standbein) et jambe libre (Spielbein).

61 «Keine Räumung um der Räumung willen», Interview de Richard von Weizsäcker, Stern, 15.02.1981.

62 Cf. Erika Fechner, Christian Gülzow, Hausbesetzungen und evangelische Kirche in Berlin-Kreuzberg. Dokumentation, Berlin, Evangelische Kirche, 1981. 
reprennent. Le 22 septembre 1981, l'évacuation de huit immeubles (dont le «Bobby Sands Haus » à Tiergarten, du nom d'un combattant de l'IRA mort d'une grève de la faim) dégénère. Au cours des affrontements qui s'ensuivent, un étudiant de 18 ans, Klaus-Jürgen Rattay, est accidentellement fauché par un bus ${ }^{(63)}$. Le congrès plénier des autonomes élabore alors un plan stratégique de barricades dans Kreuzberg 36 afin d'empêcher la police d'y pénétrer ${ }^{(64)}$.

Cette date marque une césure et la fin de l'année est plus calme. Le 7 octobre 1981, le Sénat adopte une résolution, portée par le SPD, sur l'arrêt provisoire des évacuations, suivie d'un moratoire jusqu'au printemps. Le 13 novembre a lieu la dernière action d'éclat des occupa-rénovateurs. Début 1982, les conseils d'habitants de Kreuzberg appellent tout le monde à prendre position dans le débat sur les régularisations. En juillet 1982, huit immeubles occupés se rassemblent en une coopérative autogérée, le «Kiezträger SHIK» (Selbstverwaltete Häuser in Kreuzberg), et obtiennent une régularisation. Mais la ligne souple du sénateur au Logement Rastemborski se heurte à l'inflexibilité du sénateur à l'Intérieur Lummer, qui poursuit les évacuations. Cela suscite encore quelques flambées de violence, par exemple à l'été 1983 sur le Leuschnerdamm. En 1982, le programme de rénovation urbaine est redéfini, les zones de rénovation sont réduites et l'échelle change. Le 17 mars 1983, le Sénat vote «Douze principes d'une rénovation urbaine douce» (Zwölf Grundsätze zur behutsamen Stadterneuerung) ${ }^{(65)}$. Il y est question de "maintien de la substance" (Substanzerhaltung), de "caractéristiques propres à Kreuzberg» (Eigenart Kreuzbergs), d'aménagements «conformes aux besoins» (bedarfsgerecht), de "participation» (Beteiligung) et de "représentation des habitants» (Betroffenenvertretung). En septembre 1983, le Sénat conclut un accord fiduciaire avec la société immobilière STATTBAU, qui met en œuvre les légalisations et supervise l'autogestion. En mars 1984, il n'y a plus que 18 occupations illégales à Berlin-Ouest ${ }^{(66)}$. Le 25 juillet 1984, le KuKuCK (Kunst- und Kultur-Centrum Kreuzberg) d'Anhalter Straße est évacué, ce qui provoque une manifestation spontanée d'environ 1500 personnes. Trois mois plus tard, la police procède à l'évacuation du dernier immeuble à Kreuzberg, au numéro 63a, Reichenbergerstraße.

\section{Conclusion}

C'est finalement la ligne des négociateurs et des légalisations qui l'a emporté. La révolte de SO 36 a accompagné un changement politique qui, s’il avait été annoncé dès la seconde moitié des années 1970, n’avait pas été mis en place, ou alors trop lentement ${ }^{(67)}$. Comme on peut le remarquer pour d'autres mouvements sociaux ${ }^{(68)}$,

63 Cf. les documents réunis dans: Ermittlungsausschuss im Mehringhof (éd.), abgeräumt? 8 Häuser geräumt... Klaus-Jürgen Rattay tot. Eine Dokumentation, Berlin, 1981.

64 A.G. Graumacke, Autonomie in Bewegung (note 9), p. 64.

65 On trouvera le texte original dans H. Bodenschatz, Platz frei (note 25), p. 207.

66 A. Suttner, 'Beton brennt' (note 24), p. 190.

67 Cf. Bernd Drewes, Dagmar Heyne (éd.), Kaputte Stadt retten. Ergebnisse der behutsamen Stadterneuerung in Kreuzberg. Bauen in bewohnten Häusern Adalbertstr. 80, 81, 82 in Berlin Kreuzberg, Berlin, IBA, 1985.

68 Margit MAYer, «Städtische Soziale Bewegungen», in: Ansgar Klein, Hans-Josef Legrand, Thomas Leif (éd.), Neue Soziale Bewegungen, Opladen/Wiesbaden, Westdeutscher Verlag, 1998, p. 257-271. 
celui des occupa-rénovateurs s'est fait un chemin vers les institutions et a trouvé une représentation politique au sein de la «Liste alternative». Ce chemin s'est fait au détriment de la frange la plus radicale, la plus jeune et parfois la moins «berlinoise». On estime à quelques centaines de personnes le noyau radical du mouvement, sur un total d'environ 12000 membres et sympathisants. Les personnes qui ont vécu et milité ensemble entre 1979 et 1982 venaient de milieux très divers (militants de gauche radicale et autonome, étudiants, punks, alternatifs, membres des initiatives citoyennes, habitants d'origine), et ces différences sociologiques ont contribué à fracturer le mouvement autour de la question de la négociation visant à la régularisation. Certains expriment une certaine déception ${ }^{(69)}$. Un poème militant résume la situation ainsi:

«Als wir billige Wohnungen wollten,

ließen sie Häuser leerstehen,

als wir gemeinsam im Kiez leben wollten,

rissen sie ganze Blöcke nieder,

bauten in Betonkästen dorthin, verlangten sie Wuchermieten.

Als wir dagegen demonstrierten, ignorierten sie uns.

Als wir Häuser besetzten, sagten sie, wir seien Kriminelle.

Als wir damals verhandeln wollten, drohten sie mit Räumung.

Als wir protestierten, schlugen sie uns ihre Knüppel auf den Kopf, sagten sie, wir seien kriminelle Gewalttäter.

Als wir schrien, räumten sie, denn von uns geht die Gewalt aus, sagten sie, warfen sie Tränengas, denn von uns geht die Gewalt aus, sagten sie, fertigten sie Terrorurteile, denn von uns geht die Gewalt aus, sagten sie.

Wir glauben ihnen nicht mehr ${ }^{(70)}$.

Pour autant, des lieux, infrastructures et modes de vie alternatifs ont perduré à Kreuzberg, entre magasins bio, jardins d'enfants parentaux, cinémas alternatifs et formes d'économie sociale et solidaire, englobant tous les domaines de la vie. Pour qui voulait vivre à la kreuzbergeoise, il y avait même une «bible», le WestBerliner Stattbuch, répertoriant les lieux, groupes et projets alternatifs ${ }^{(71)}$. Kreuzberg est resté en partie un «autre lieu», une «hétérotopie» chère à Michel Foucault ${ }^{(72)}$. Si la rénovation douce, à

69 Dieter Hoffmann-Axtheim, Straßenschlachtung: Geschichte, Abriß und gebrochenes Weiterleben der Admiralstraße, Berlin, Nishen, 1984.

70 Cité dans H. Bodenschatz, Platz frei (note 25), p. 204.

71 Arbeitsgruppe WestBerliner Stattbuch (éd.), WestBerliner Stattbuch, Berlin, 1978 (1 $1^{\mathrm{re}}$ éd.).

72 C'est l'IBA qui publie en 1984 - avec l'autorisation de l'auteur peu de temps avant sa mort - le discours prononcé en 1967 sur les «autres lieux». Michel FoucAult, «Andere Räume», in: IBA (éd.), Idee Prozeß Ergebnis (note 23). 
échelle humaine et participative est devenue un attendu de toute politique urbaine, les luttes n'en ont pas cessé pour autant ${ }^{(73)}$. Après la chute du Mur, une nouvelle vague d'occupations a lieu, à Berlin-Est cette fois (Prenzlauer Berg et Friedrichshain) ${ }^{(74)}$. Ces vingt dernières années, la prise de conscience des effets de la gentrification, du rôle que jouent les pouvoirs publics dans l'accompagnement du changement social et l'éviction des couches socialement faibles hors des centres-villes n'est pas sans rappeler les débats des années 1970 et 1980. La ville reste un terrain d'étude primordial pour une géographie sociale radicale ${ }^{(75)}$.

\section{Résumé}

Berlin-Ouest a rassemblé dans l'après-1968 un important milieu alternatif, marqué par une subculture et des actions collectives. Cette politique au quotidien est attachée à des lieux précis, par exemple le quartier de Kreuzberg SO 36. Le mouvement des occupations d'immeubles au tournant des années 1970 et 1980 répondait à un problème social et aux manquements de la politique en matière d'urbanisme menée jusque-là. Les occupants ont repensé la ville comme un ensemble d'espaces multiples, produits par des usages sociaux soumis à des rapports de pouvoir. Malgré d'importantes dissensions internes, ils partageaient, outre une sociologie et des modes de vie communs, un répertoire politique, des formes d'action et un langage radical. Par leurs actions parfois spectaculaires, ils ont contribué à «faire la ville».

\section{Zusammenfassung}

West-Berlin stand im Zuge von 1968 für eine aktive alternative Szene, mit ihren Subkulturen und kollektiven Mobilisierungen. Diese Politik von unten verortete sich in bestimmten Vierteln, beispielsweise Kreuzberg SO 36. In den 1970er und 1980er Jahren war die Hausbesetzerbewegung eine Antwort aufeine soziale Wohnungsnot und auf die Defizite der Stadterneuerungspolitik in der Nachkriegszeit. Den Hausbesetzern galt die Stadt als ein multipler und verflochtener Raum, in dem soziale Praktiken stattfanden

73 Hartmut HäUssermanN, «Segregation - Partizipation - Gentrifikation. Zur Bedeutung von kulturellem Kapital in der Stadterneuerung», in: Jens S. Dangschat, Alexander Hamedinger (éd.), Lebensstile, soziale Lagen und Siedlungsstrukturen, Hanovre, Akademie für Raumforschung und Landesplanung, 2007, p. 161-181.

74 Susan Arndt (éd.), Berlin, Mainzer Straße: “wohnen ist wichtiger als das Gesetz”, Berlin, Basis-Druck, 1992; Hartmut Häussermann, Andrej Holm, Daniela Zunzer, Stadterneuerung in der Berliner Republik, Modernisierung in Berlin-Prenzlauer Berg, Opladen, Leske + Budrich, 2002; Wolfram Keмpe, "Aufbruchstimmung in Prenzlauer Berg. Hausbesetzungen in der Dunckerstrasse», in: Bernt Roder, Bettina Tacke (éd.), Prenzlauer Berg im Wandel der Geschichte. Leben rund um den Helmholtzplatz, Berlin, be.bra Verlag, 2004, p. 223-241. Dans les années 1980, des occupations avaient aussi eu lieu à Berlin-Est, mais selon des modalités différentes qu'à l'Ouest. Voir Christian HaLbrock, "Vom Widerstand zum Umbruch: die oppositionelle Szene in den 80er Jahren", in: ibid., p. 98-124; Wolfgang KIL, «Kaum Konfrontation, eher Unterwanderung», in: Schlusche et al. (éd.), Stadtentwicklung (note 19), p. 190-199.

75 Cf. David Harvey, Villes rebelles. Du droit à la ville à la révolution urbaine, trad. O. Demange, Paris, Éditions Buchet/Chastel, 2015; Anne Clerval, Antoine Fleury, Julien Rebotier, Serge Weber (éd.), Espace et rapports sociaux de domination, Rennes, PUR, 2015. 
und Machtverhältnisse bestanden. Trotz zahlreicher interner Unstimmigkeiten war diese Bewegung von gemeinsamen soziologischen, weltanschaulichen, politischen und diskursiven Merkmalen gekennzeichnet. Durch ihre teilweise spektakulären Mobilisierungen hat die Hausbesetzerbewegung «Stadt gemacht». 